\title{
ESTIMATIVA DE CONSTITUINTES OPTICAMENTE ATIVOS DA ÁGUA POR SENSORIAMENTO REMOTO ORBITAL
}

\author{
ESTIMATION OF OPTICALLY ACTIVE CONSTITUENTS OF WATER BY REMOTE ORBITAL SENSING
}

\begin{abstract}
RESUMO
O trabalho teve como objetivo avaliar o uso potencial de imagens LISSIII/Resourcesat-1 na estimativa de constituintes opticamente ativos da água do reservatório Passo Real. Com as imagens de satélite utilizadas para a análise da refletância da água foi possível a extração de informações em pixels com resolução espacial de 23,5 metros. A seleção de imagens foi feita a partir da disponibilidade de cenas sem presença de nuvens em datas mais próximas à realização dos trabalhos de campo. Os processamentos das imagens para correção dos efeitos atmosféricos e transformação dos números digitais em valores de reflectância foram realizados no software ENVI 5.0 As espacializações dos dados limnológicos obtidos in situ foram realizadas por interpolação no software Spring 4.3 .3 e as espacializações dos dados estimados por satélite foram gerados por fatiamento da imagem gerada pela inserção das equações obtidas nos modelos. A partir do conjunto de dados disponível foi possível produzir modelos capazes de estimar o total de sólidos em suspensão, a turbidez e a transparência da água com apenas uma única banda espectral, a banda 3 (620 a $680 \mathrm{~nm}$ ) do sensor LISSIII/Resourcesat-1. Com a aplicação da técnica de razão de bandas espectrais foi possível gerar um modelo para estimativa da concentração de clorofila- $a$.
\end{abstract}

Palavras-chave: variáveis limnológicas, reflectância, imagens LISS-III.

\section{ABSTRACT}

The objective of this study was to evaluate the potential use of LISS-III / Resourcesat-1 images in the estimation of optically active constituents of water in the Passo Real reservoir. With the satellite images used for the analysis of the water reflectance, it was possible to extract information in pixels with spatial resolution of 23.5 meters. The selection of images was made from the availability of scenes without clouds at the closers days to the field work. The processing of the images to correct the atmospheric effects and the transformation of the digital numbers into reflectance values were performed in the software ENVI 5.0 limnological data spatialization obtained in situ were carried out by interpolation in the software Spring 4.3.3 and the spatializations of the data estimated by satellite were generated by slicing the image generated by the insertion of equations obtained in the models. From the available data set it was possible to produce models capable of estimating total suspended solids, turbidity and water transparency with only a single spectral band, band 3 ( 620 to $680 \mathrm{~nm}$ ) of the LISS-III / Resourcesat-1. With the application of the spectral band ratio technique, it was possible to generate a model to estimate the chlorophyll-a concentration.

Keywords: limnological variables, reflectance, LISS-III images.

\author{
Felipe Correa dos Santos ${ }^{a}$ \\ Waterloo Pereira Filho ${ }^{a}$ \\ ${ }^{\text {a } U n i v e r s i d a d e ~ F e d e r a l ~ d e ~ S a n t a ~ M a r i a ~}$ \\ (UFSM), Santa Maria, RS, Brasil
}

DOI: $10.12957 /$ geouerj.2020.38068

Correpondência:

felipecorrea_rs@hotmail.com

Recebido em: 30 out. 2018

Revisado em: 7 jan. 2020

Aceito em: 6 out. 2020 


\section{INTRODUÇÃO}

O sensoriamento remoto é utilizado como ferramenta em estudos de estimativas de parâmetros de avaliação da qualidade da água, devido a possibilidade de acompanhar a variação espacial e temporal da composição da água e investigar a origem e o deslocamento de substâncias específicas em suspensão ou dissolvidas na água (BARBOSA, 2005; RUDORFF, 2006; JENSEN, 2009). As estimativas de parâmetros de avaliação da qualidade da água são baseadas nas relações entre variáveis limnológicas e valores de reflectância obtidas em uma ou mais bandas espectrais.

A atual disponibilidade de dados espectrais oriundos de imagem de satélite trouxe novas perspectivas para as análises em ambientes aquáticos, pois são capazes de fornecer para cada pixel (elemento de resolução espacial), grande quantidade de informação espectral. Essa característica possibilita a distinção de diferentes componentes do ambiente aquático, visto que as variações na composição de substâncias opticamente ativas na água provocam diferentes padrões de absorção e espalhamento da radiação eletromagnética, passíveis de serem detectadas por sensores (ENNES, 2008). As variações na resposta espectral da água causadas pela presença dessas substâncias, têm sido explicadas por diversos autores (GOODIN et al., 1993; MOBLEY, 1994; KIRK, 1994; HAN 1997; KAMPEL; NOVO, 2005; FOLKESTAD et al., 2007; NOVO et al., 2007; LOBO, 2009).

Em águas continentais, os constituintes opticamente ativos da água são passíveis de detecção por técnicas de sensoriamento remoto (KAMPEL; NOVO, 2005). Dentre estas substâncias e/ou partículas se destacam o total de sólidos em suspensão e os pigmentos fotossintéticos como a clorofila-a, presente nos organismos fitoplanctônicos aquáticos (MOBLEY, 1994). Estes elementos influenciam na turbidez e transparência da água e imprimem características únicas nos dados de sensoriamento remoto, o que permite a identificação dos constituintes opticamente ativos e o consequente monitoramento do ambiente aquático.

Para a verificação do estado de qualidade da água e dos fatores que afetam a superfície do ambiente aquático são utilizados métodos de amostragens tradicionais que requerem medições in situ, ou seja, a coleta de amostras de água em campo para análise laboratorial subsequente. Os métodos de amostragem tradicionais possibilitam obtenção de medições de maior precisão, no entanto, requerem tempo e resultam em maiores custos para sua realização, já que a espacialização dos dados, por interpolação, exige a alocação de um grande número de pontos amostrais para que seja estatisticamente significativa. 
Técnicas de sensoriamento remoto, no entanto, podem ser utilizadas para detectar as propriedades ópticas dos constituintes opticamente ativos da água, sanando as dificuldades de amostragem em campo e ainda contribuem para a melhoria da informação espacial e temporal sobre as ocorrências de altas concentrações de algas (BRANDO; DEKKER, 1993; HAN; RUNDQUIST, 1996; KUTSER, 2004). A utilização de imagens de sensores remotos e de dados obtidos in situ para estudar florações de algas é baseada no fato de que os pigmentos de algas afetam a cor da massa de água.

Diversos produtos de sensoriamento remoto com diferentes resoluções temporais, espaciais, espectrais ou radiométricas são disponibilizados gratuitamente. Dentre estes cita-se as imagens dos satélites da série IRS (Indian Remote Sensing satellite), mais especificamente do sensor LISS-III (Linear Imaging Self-Scanner III), a bordo do satélite indiano ResourceSat-1. No Brasil, os dados do sensor LISS-III são distribuídos gratuitamente pelo Instituto Nacional de Pesquisas Espaciais (INPE) com o intuito de viabilizar dados com características similares às apresentadas pelo sensor TM/Landsat para que fosse garantida a continuidade de trabalhos que já vinham sendo conduzidos pelo público acadêmico e corporativo. Berra et al. (2013) e Silva et al. (2013) recomendam a utilização do sensor LISS-III como complemento e substituição aos dados TM/Landsat 5, levando em consideração as semelhanças entre as resoluções dos sensores e, por consequência, a reflectância.

O potencial de utilização de imagens LISS-III é explorado para monitoramento de ambientes aquáticos e diversas pesquisas utilizam de seus produtos para avaliar a qualidade da água. Somvanshi et al (2012) utilizou dados de imagens LISS-III para estimar e mapear parâmetros de qualidade da água a partir de modelos de regressões lineares. Dados de reflectância de imagens LISS-III utilizados em conjunto com dados de espectrorradiômetros foram importantes para compreensão do funcionamento espaço-temporal de reservatórios estudados realizados por Thiemann e Kaufmann (2000). Valério e Kampel (2013) utilizaram imagens LISS-III para a caracterização espectral da pluma do Rio Paraíba do Sul e as reflectâncias derivadas das diferentes bandas possibilitaram descrever qualitativamente a distribuição espacial dos constituintes opticamente ativos presentes na área de estudo.

Inserido neste contexto, este estudo propõe o uso de metodologias relacionadas ao sensoriamento remoto para o estudo de variáveis limnológicas, bem como a identificação e mapeamento de constituintes opticamente ativos no reservatório Passo Real. As técnicas utilizadas serão pautadas em relações e estimativas do total de sólidos em suspensão, turbidez e transparência da água com imagens do sensor LISS-III e aplicação da técnica de razão de bandas 
espectrais com o intuito de identificar a concentração de clorofila-a. Como resultados espera-se obter informações relacionadas presença e concentração de constituintes opticamente ativos da água e a possibilidade de monitoramento do ambiente aquático utilizando produtos e técnicas de sensoriamento remoto orbital.

Diante disso, este trabalho tem como objetivo de avaliar o uso potencial de imagens LISSIII/Resourcesat-1 na estimativa de constituintes opticamente ativos da água do reservatório Passo Real.

\section{MATERIAIS E MÉTODOS}

Para a realização da presente pesquisa no reservatório Passo Real foram utilizadas metodologias distribuídas em três fases: coleta de água e obtenção de dados limnológicos em campo, determinação de variáveis em laboratório de limnologia e obtenção de dados espectrais em laboratório de processamento de imagens. Salienta-se que a presente pesquisa está relacionada a um projeto financiado pelo CNPq no 478961/2010-3 (Variação espectral e limnológica nos reservatórios em cascata no rio Jacuí - Rio Grande do Sul).

\section{Coletas in situ de dados limnológicos}

Para a obtenção dos dados de clorofila-a no reservatório Passo Real foram realizadas coletas de amostras de água em campo e posterior determinação de dados em laboratório de limnologia. Para a realização dos trabalhos de campo no reservatório Passo Real, Pereira Filho et al. (2010) estabeleceram 31 estações amostrais que contemplam as diferenças espectrais encontradas no reservatório (Figura 1), porém se ressalta que nem todos os pontos foram coletados em campo nos meses analisados nesta pesquisa. Em 15 de setembro de 2012 foram coletadas 8 amostras (pontos 8, 10, 12, 16, 20, 28, 29 e 30) e em 23 de janeiro de 2013 foram coletadas 11 amostras (pontos 4, 5, 8, 10, 12, 16, 20, 28, 29, 30 e 31 .

Para a realização das coletas de dados em campo foi utilizada a infraestrutura pertencente ao Laboratório de Geotecnologias da Universidade Federal de Santa Maria, que dispõe de automóvel, barco e motor de popa, entre outros equipamentos e materiais para coleta e análise da amostra de água em laboratório. 
Figura 1. Localização dos pontos amostrais no reservatório Passo Real

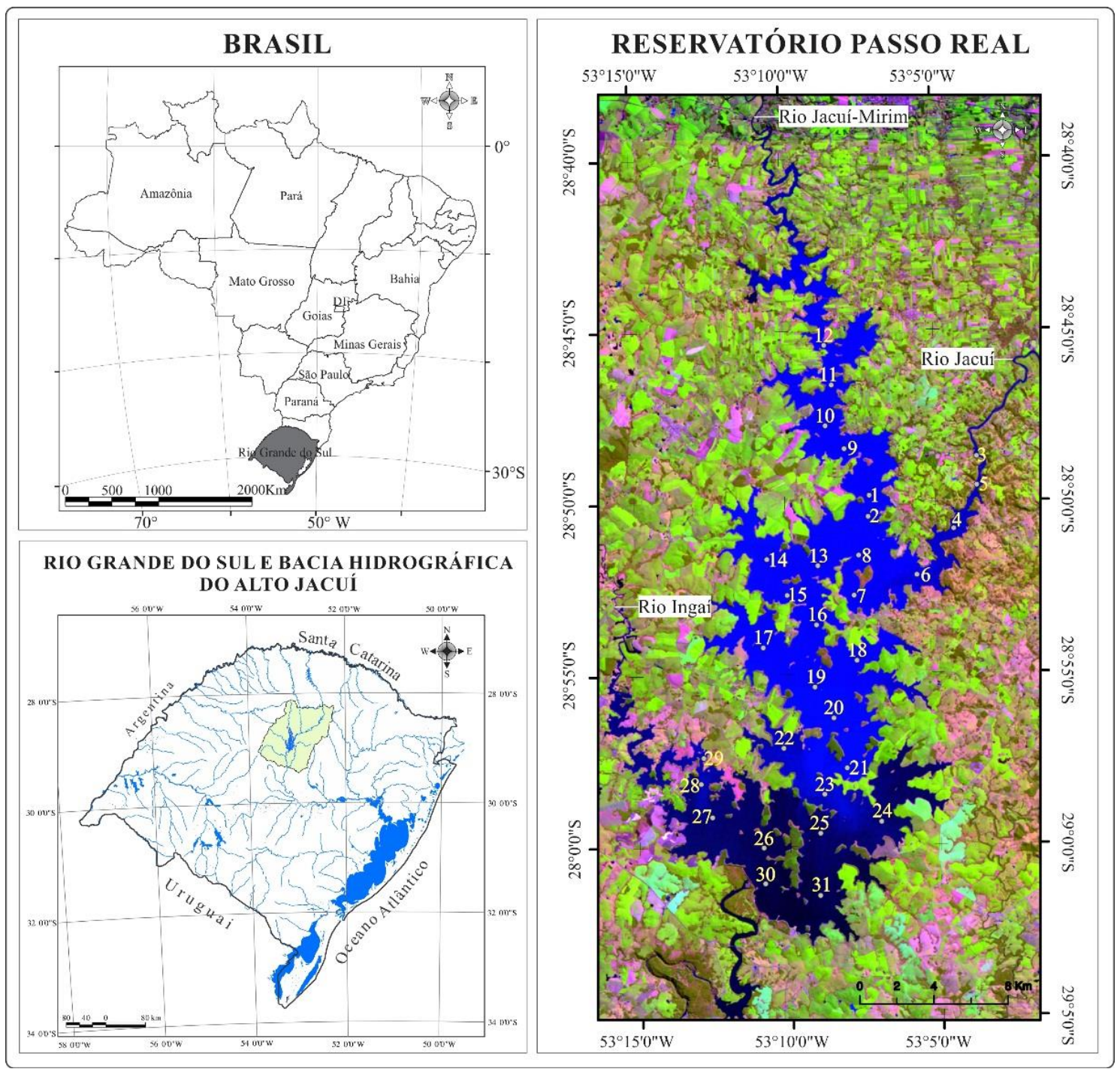

Fonte: TONIOLO, et al. (2012).

Para a localização das estações amostrais do reservatório foi utilizado um aparelho GPS Garmin Etrex de precisão de 5 metros, com as coordenadas geográficas das estações previamente armazenadas no aparelho. A transparência da água foi medida em campo com a utilização de um Disco de Secchi com diâmetro de $30 \mathrm{~cm}$. Os valores de turbidez e oxigênio dissolvido foram obtidos com o mergulho da sonda multiparâmetros Horiba modelo U53.

Para as variáveis determinadas em laboratório foi realizada a coleta da água em cada ponto amostral em subsuperfície, na profundidade aproximada de $30 \mathrm{~cm}$, com a utilização de recipientes numerados e com um litro de capacidade, que após a coleta foram mantidos em recipientes térmicos e escuros até a chegada ao Laboratório de Limnologia. Em laboratório foram determinadas as variáveis: Total de Sólidos em Suspensão (TSS) e concentração de clorofila-a (Chl-a). 
A determinação dos valores de TSS em laboratório foi realizada conforme apresentado em APHA (2005). Para a filtragem foi utilizada bomba de vácuo e filtros de celulose com 0,45 $\mu \mathrm{m}$ de porosidade e $47 \mathrm{~mm}$ de diâmetro. Os filtros foram secados previamente por 24 horas em estufa a uma temperatura de $50^{\circ} \mathrm{C}$, a fim de eliminar a umidade. Posteriormente à secagem, os filtros foram pesados em balança analítica para obtenção do peso inicial. Após a filtragem da água, os filtros retornaram à estufa, onde permanecem por 24 horas a $50^{\circ} \mathrm{C}$ novamente. Após, obtevese o peso final com a pesagem dos filtros na mesma balança e determinou-se a concentração de total de sólidos em suspensão em mg/L com base na Equação 1.

$$
\mathrm{TSS}=\left(\frac{P f-P i}{V}\right) \cdot 1000
$$

(Equação 1)

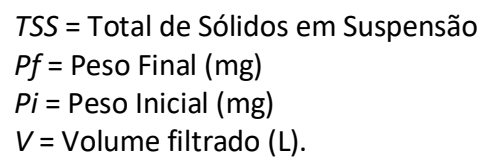

A clorofila- $a$ foi determinada em laboratório conforme método apresentado por Yunes e Araújo [s/d] com base em Mackinney (1941), Paranhos (1996) e Chorus e Bartram (1999). Para a determinação de clorofila- $a$ os procedimentos de filtragem e extração de pigmentos foram realizados sempre sob fraca iluminação, para inibir a realização da fotossíntese. Para a filtragem foram utilizados filtros de fibra de vidro. Na sequência os filtros foram removidos e colocados sobre um papel absorvente para retirar a umidade, enrolados em papel alumínio e congelados, para a preservação dos pigmentos até sua extração. Para a extração dos pigmentos os filtros foram colocados dentro de frascos com $10 \mathrm{ml}$ de metanol absoluto e depois de tampados foram deixados em refrigeração (4ㅇ) por 24 horas. Para a leitura no espectrofotômetro foram retirados os filtros da refrigeração, retirado o sobrenadante com uma pipeta e colocadas as amostras nas cubetas do espectrofotômetro. Mackinney (1941) explica que a absorbância da clorofila- $a$ ocorre em $663 \mathrm{~nm}$ e a turbidez das células das cianobactérias em $750 \mathrm{~nm}$. Para o cálculo da concentração de clorofila- $a$ em $\mu \mathrm{g} / \mathrm{L}$ foi utilizada a Equação 2 em que são levados em consideração as medições do espectrofotômetro, o volume de pigmentos extraídos e o volume filtrado.

$$
\operatorname{Chl} a=\frac{\operatorname{Abs}(663-750) \times 12,63 \times \mathrm{VE} \times 1000}{\mathrm{VF}}
$$


Aquisição de imagens LISS-III

As imagens de satélite utilizadas para a análise temporal da refletância da água foram produzidas pelo satélite ResourceSat-1, sensor LISS-III, a partir das quais é possível a extração de pixels puros em função da resolução espacial de 23,5 metros. O sensor apresenta resolução temporal de 24 dias, opera em quatro faixas espectrais (verde - 520 a $590 \mathrm{~nm}$, vermelho - 620 a $680 \mathrm{~nm}$, infravermelho próximo - 770 a $860 \mathrm{~nm}$ e infravermelho médio - 1550 a $1700 \mathrm{~nm}$ ) e possui resolução radiométrica de 7 bits (NRSA, 2004). A seleção de imagens foi feita a partir da disponibilidade de cenas sem presença de nuvens em datas mais próximas à realização dos trabalhos de campo, conforme apresentado na Quadro 1.

Quadro 1. Datas dos trabalhos de campo no reservatório Passo Real e das imagens de satélite ResourceSat-1/LISS-III.

\begin{tabular}{|l|l|l|}
\hline Data de coleta de dados in situ & $\begin{array}{l}\text { Data da imagem selecionada } \\
\text { (reflectância) }\end{array}$ & $\begin{array}{l}\text { Defasagem entre data dos dados in } \\
\text { situ e reflectância (em dias) }\end{array}$ \\
\hline 15.09 .2012 & 05.09 .2012 & -10 \\
\hline 23.01 .2013 & 27.01 .2013 & +4 \\
\hline
\end{tabular}

As imagens do satélite ResourceSat-1 foram adquiridas gratuitamente no catálogo do Instituto Nacional de Pesquisas Espaciais (http://www.dgi.inpe.br/CDSR). A área referente ao reservatório Passo Real está compreendida na órbita/ponto 326/099.

\section{Processamentos de imagens LISS-III}

Uma etapa anterior ao processamento de imagens é o pré-processamento, que consiste na preparação do dado para ser interpretado. Nessa fase ocorreu a correção atmosférica e a correção radiométrica das imagens de satélite. Na fase de processamento ocorreu a classificação digital das imagens.

\section{Correção dos efeitos atmosféricos}

O processamento das imagens foi realizado no software ENVI 5.0. As bandas foram corrigidas dos efeitos da atmosfera pelo método da Subtração do Pixel Escuro (Dark-Object Subtraction), proposto por Chavez Junior (1988). Segundo a proposta deste método, em toda e qualquer cena e em qualquer banda espectral, existem pixels que deveriam assumir o valor " 0 ", uma vez que estes podem não ter recebido radiação incidente. No caso de sombras na região do visível, idealmente o sistema de imageamento não deve detectar qualquer radiação nesses locais e um valor "0" de número digital deve ser atribuído a estes pixels. No entanto, por causa de efeitos de dispersão na atmosfera, estas áreas sombreadas não aparecerão completamente escuras na imagem. Dessa, forma, a correção consistiu em identificar no histograma de cada banda espectral, quais 
quantidades de números digitais devem ser subtraídas de cada imagem como um todo, considerando que a interferência atmosférica é uniforme em toda a cena.

\section{Conversão de Números Digitais para valores físicos de Radiância e Reflectância}

Os valores em números digitais da imagem foram transformados em valores de radiância espectral e em seguida convertidos em reflectância de superfície conforme métodos descritos em Markham e Barker (1986). A conversão de Números Digitais (ND) das imagens para valores de parâmetros físicos como Radiância e/ou Reflectância permite a caracterização espectral de objetos, bem como permite a elaboração de cálculos que incluem dados de diferentes bandas espectrais ou de diferentes sensores. Este processo exigiu o conhecimento de algumas características do sensor que gera a imagem e das condições ambientais nas quais as imagens foram geradas (CHANDER, 2016) obtidos nos metadados das respectivas imagens e que estão presentes no Tabela 1.

Tabela 1. Dados utilizados na conversão dos números digitais das imagens LISS-III/Resourcesat-1 em valores de radiância aparente

\begin{tabular}{|c|c|c|c|c|c|}
\hline Banda & $L M I N_{\lambda}$ & $L M A X_{\lambda}$ & $D_{\max }$ & $L M A X_{\lambda} / D \max$ & $\operatorname{Esun}_{\lambda}$ \\
\hline 2 & 0 & 12,06400 & 255 & 0,0473098 & 1846,77 \\
\hline 3 & 0 & 15,13100 & 255 & 0,0593370 & 1575,5 \\
\hline 4 & 0 & 15,75700 & 255 & 0,0617922 & 1087,34 \\
\hline 5 & 0 & 3,40000 & 255 & 0,0133568 & 236,651 \\
\hline
\end{tabular}

O primeiro processamento consistiu em converter os Números Digitais em valores de radiância espectral usando parâmetros de calibração obtidos em missões de calibração antes do lançamento, segundo a Equação 3.

$$
L_{\lambda}=\operatorname{Lmin}_{\lambda}+\left(\frac{L M A X_{\lambda}-L M I N_{\lambda}}{Q_{\text {calmax }}}\right) Q_{C A L}
$$

$L_{\lambda}=$ Radiância espectral,

$Q_{C A L}=$ Número digital a ser convertido,

$Q_{\text {calmax }}\left(D_{\max }\right)=$ Número digital máximo (dependente da resolução radiométrica do sensor),

$\operatorname{LMIN}_{\lambda}=$ Radiância espectral minima,

$L M A X_{\lambda}=$ Radiância espectral máxima.

Após a conversão para radiância, os dados adquiridos foram convertidos para Fator de Reflectância Bidirecional Aparente (FRB), por meio da Equação 4. 


$$
\rho_{\lambda}=\frac{\pi * L_{\lambda} * d^{2}}{\operatorname{Esun}_{\lambda} * \cos \theta} \quad \text { (Equação 4) }
$$

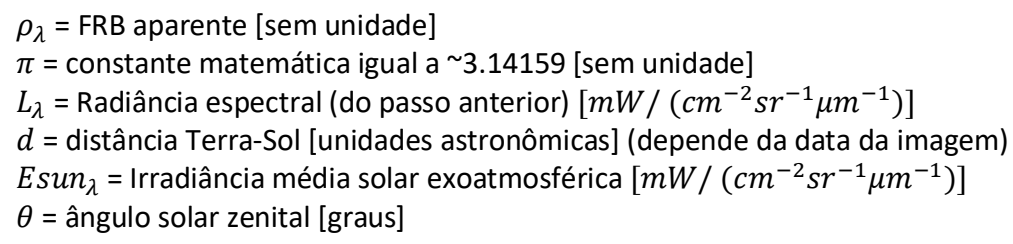

\section{Razão de bandas}

A técnica de razão de bandas foi aplicada nos dados de reflectância sensor LISS-III/ResourceSat-1 nas imagens de 05 de setembro de 2012 e 27 de janeiro de 2013 e seus resultados foram correlacionados com clorofila-a. Esta técnica é determinada por meio da razão entre dois valores de reflectância. Dessa forma foi utilizada a banda 4 (infravermelho próximo) do sensor LISS-III/ResourceSat-1 como numerador e a banda 3 (vermelho) como denominador para obtenção dos valores no software Envi.

\section{Relação entre dados limnológicos e espectrais}

Dados de amostras pontuais de reflectância foram consultados nas imagens a partir das coordenadas geográficas dos pontos de coleta do reservatório. Para tanto, foram extraídos valores de reflectância dos pixels mais próximos das áreas de interesse e com menor interferência de alvos vizinhos. A técnica de razão de bandas espectrais foi aplicada com o intuito de verificar a relação com dados de concentração de clorofila- $a$.

A etapa metodológica seguinte correspondeu à análise integrada dos dados limnológicos e espectrais. Com os dados limnológicos e espectrais organizados em tabelas no software Microsoft Office Excel foram realizados testes estatísticos de regressão para interpretar e comparar os dados estudados. Nas análises de regressão, a reflectância espectral foi considerada a variável independente e o constituinte opticamente ativo da água foi considerado variável resposta e obteve-se assim uma equação matemática que representa o comportamento destes dados, além de um coeficiente de determinação $\left(R^{2}\right)$, que representa o nível da variância que o modelo consegue explicar.

As equações obtidas nos modelos de regressão foram aplicadas nas imagens LISS-III utilizadas para a geração dos modelos. Para isso, utilizou-se o software Envi 5.0 com o intuito de testar a estimativa dos constituintes opticamente ativos da água a partir dos dados espectrais das bandas das imagens de satélite.

As medidas das variáveis limnológicas obtidas pelas equações nas imagens de satélite foram fatiadas em 20 classes temáticas, da mesma forma que os resultados obtidos in situ. Para as variáveis transparência da água e clorofila- $a$ as 20 classes temáticas foram definidas e ponderadas de acordo com os estados tróficos 
definido por Carlson (1977) e modificados por Lamparelli (2004). A partir desses resultados, pode-se comparar a espacialidade das variáveis obtidas in situ e as estimadas por imagem de satélite.

\section{RESULTADOS E DISCUSSÃO}

O total de sólidos em suspensão obteve maior correlação com a reflectância da banda 3 do sensor LISSIII. O modelo de regressão apresentou coeficiente de determinação 0,65 , considerado de intensidade regular, porém significativo com $95 \%$ de confiança. O modelo de regressão apresentou ajuste linear dos dados e a equação matemática é apresentada na Figura 2.

Figura 2. Diagrama de dispersão entre o total de sólidos em suspensão e a reflectância da banda 3 do sensor LISS-

III/ResourceSat-1.

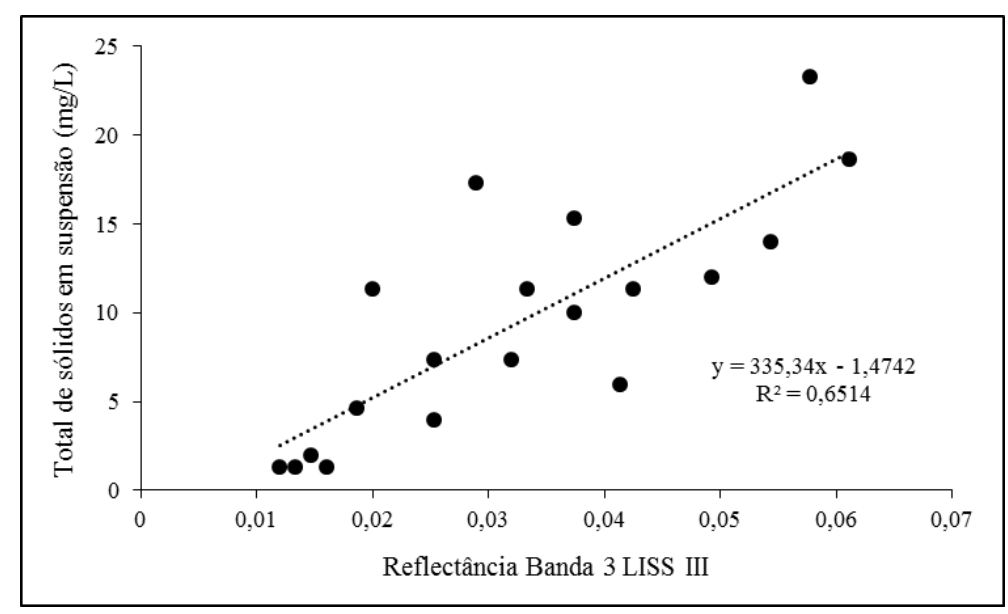

Dados semelhantes são encontrados em estudo realizado por Wani et al (1996) em que relatam a existência de uma correlação positiva entre a concentração de total de sólidos em suspensão da água Lago Dal no norte da Índia e a reflectância da banda 3 do sensor LISS-III.

A Figura 3 apresenta a espacialização do total de sólidos em suspensão obtido in situ no reservatório Passo Real e estimado pela reflectância da banda 3 do sensor LISS-III/ResourceSat-1. Concentrações de sólidos em suspensão foram observadas em todo reservatório, ocorrendo apenas variações de acordo com as particularidades de cada setor e o período estudado. Os registros de TSS foram mais elevados em setembro de 2012 comparados com os dados de janeiro de 2013. Ao observar os registros de TSS na região central do reservatório obtidos na imagem de 27 de janeiro de 2013 nota-se um avanço da carga de sólidos em suspensão à jusante quando compara-se com o local de mais alta concentração de TSS no dia 23 de Janeiro de 2013. 
Observa-se nos mapas da Figura 3 proximidade entre os dados estimados e medidos em campo, principalmente em janeiro de 2013. No mês de setembro de 2012 os valores estimados foram semelhantes a partir dos setores centro-norte do reservatório, correspondentes às entradas dos rios Jacuí e Jacuí-mirim. Próximo ao dique e em algumas partes da região centro-sul do reservatório os valores de TSS foram subestimados em comparação aos dados obtidos in situ. Este resultado era esperado visto que o modelo de regressão aplicado na imagem apresentou coeficiente de determinação 0,65. Em uma abordagem geral, o modelo linear capturou a transição de montante para jusante da concentração de TSS, em concordância com os dados medidos em campo.

Figura 3. Espacialização do total de sólidos em suspensão obtido in situ no reservatório Passo Real e estimado pela reflectância da banda 3 do sensor LISS-III/ResourceSat-1.

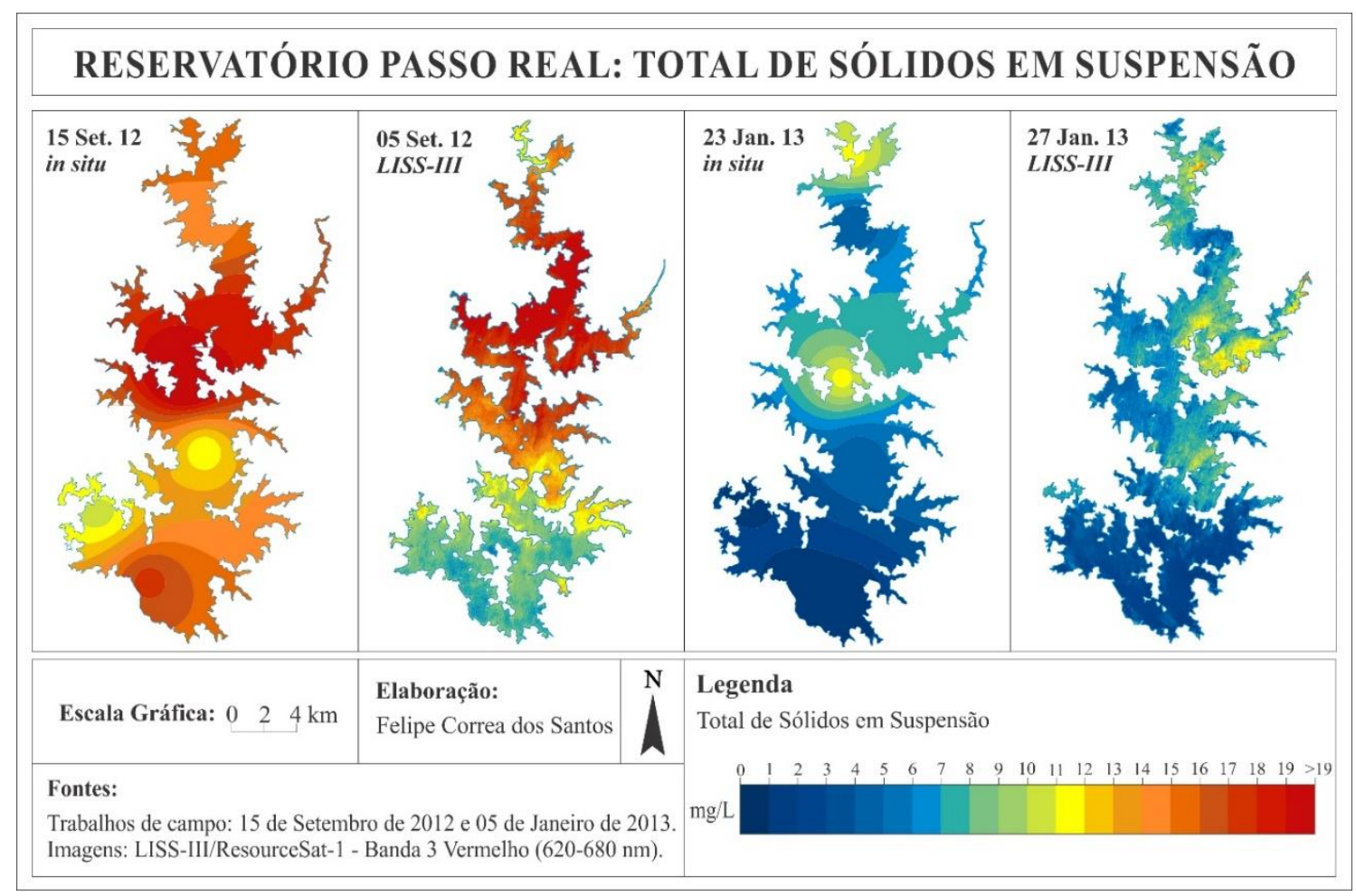

Os valores estimados de total de sólidos em suspensão da água são compatíveis aos relatados na literatura para águas de ambientes aquáticos continentais (autores), onde dados in situ são utilizados para validação de valores estimados por imagens de satélite (CHOUBEY; SUBRAMANIAN, 1992; LOBO et al, 2014; AVDAN et al, 2019).

A turbidez da água obteve maior correlação com a reflectância da banda 3 do sensor LISS-III. O modelo de regressão apresentou coeficiente de determinação superior a 0,92, considerado de intensidade muito forte e significativo com $95 \%$ de confiança. O modelo de regressão apresentou ajuste linear dos dados e a equação matemática que explica o relacionamento é apresentada na Figura 4. 
Figura 4. Diagrama de dispersão entre a turbidez da água e a reflectância da banda 3 do sensor LISS-III/ResourceSat-1.

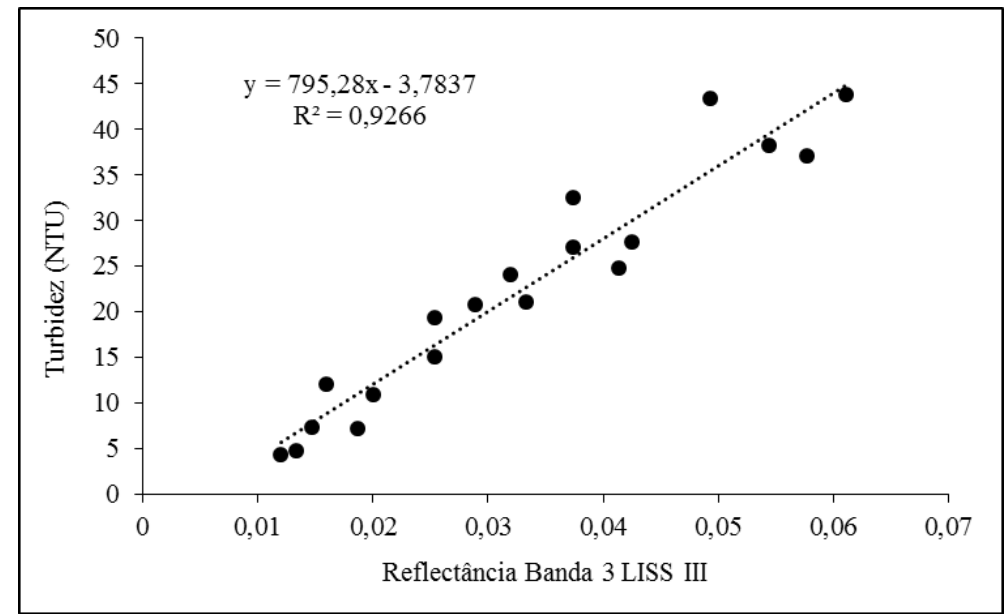

Estudo realizado por Sheela et al (2012) nos sistemas aquáticos Akkulam e Veli localizados no sudoeste da Índia apresenta modelos de regressão com significativo ajuste linear entre bandas individuais de imagens LISS-III para estimativa da turbidez.

A Figura 5 apresenta a espacialização da turbidez obtida in situ no reservatório Passo Real e estimada pela reflectância da banda 3 do sensor LISS-III/ResourceSat-1.

Figura 5. Espacialização da turbidez da água obtida in situ no reservatório Passo Real e estimada pela reflectância da banda 3 do sensor LISS-III/ResourceSat-1.

\begin{tabular}{|l|l|l|l|}
\hline \multicolumn{3}{|c|}{ RESERVATÓRIO PASSO REAL: TURBIDEZ } \\
\hline in set. 12
\end{tabular}


Observa-se que houve consistência entre os dados estimados e medidos em campo nas duas datas analisadas. Em ambos os métodos de aquisição de dados pode-se observar que a turbidez da água do reservatório em setembro de 2012 foi mais elevada se comparada com a do mês de janeiro de 2013. Em ambas as datas, o setor de mistura das entradas dos rios Jacuí e Jacuí-Mirim apresentou os registros mais elevados de turbidez e o setor próximo ao dique se caracterizou como área do reservatório de baixa turbidez.

O modelo de regressão aplicado na imagem apresentou coeficiente de determinação superior 0,92, o que contribuiu a elevada concordância dos dados obtidos por imagem de satélite com os valores de turbidez da água medidos em campo.

Aghighi et al (2008) testou a utilidade dos dados LISS-III para o mapeamento da turbidez da água na Baía de Gorgan, a sudeste do Mar Cáspio, localizada no norte do Irã. Após a correção dos erros geométricos e radiométricos, os dados resultantes da radiância foram usados para examinar as correlações entre os dados de turbidez da água detectados remotamente e in situ. Os resultados da pesquisa mostraram boas relações entre a turbidez e os dados espectrais tornando-se possível o mapeamento e monitoramento da turbidez da água.

A transparência da água medida em campo com o disco de Secchi obteve maior correlação com a reflectância da banda 3 do sensor LISS-III. O modelo de regressão apresentou coeficiente de determinação 0,86, considerado de intensidade forte e significativo com 95\% de confiança (Figura 6).

Figura 6. Diagrama de dispersão entre a transparência da água e a reflectância da banda 3 do sensor LISS-III/ResourceSat-1.

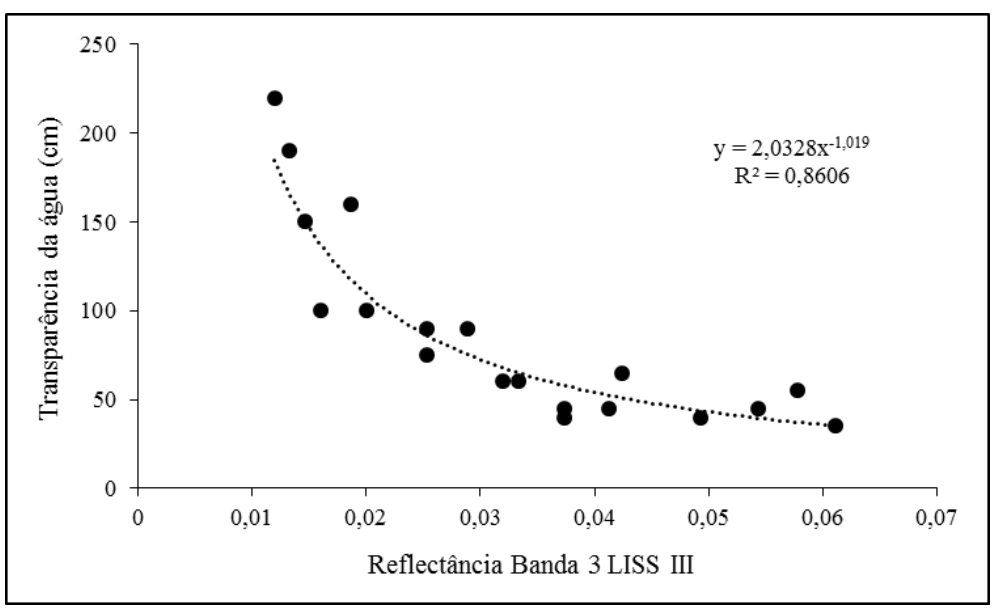

Pesquisa realizada por Hellweger et al. (2004) relata que a reflectância na faixa do vermelho foi a que melhor correlacionou com dados de transparência da água. Wu et al. (2008) expõe que a faixa do vermelho em dados Landsat 5 TM apresentou correlações negativas com dados de transparência. Procedimentos 
semelhantes foram aplicados por McCullough (2012) para monitoramento remoto da transparência da água com imagens TM de lagos em Maine, localizada no nordeste dos Estados Unidos.

A Figura 7 apresenta a espacialização da transparência da água observada in situ no reservatório Passo Real e a estimada pela reflectância da banda 3 do sensor LISS-III/ResourceSat-1.

Figura 7. Espacialização da transparência da água obtida in situ no reservatório Passo Real e estimada pela reflectância da banda 3 do sensor LISS-III/ResourceSat-1.

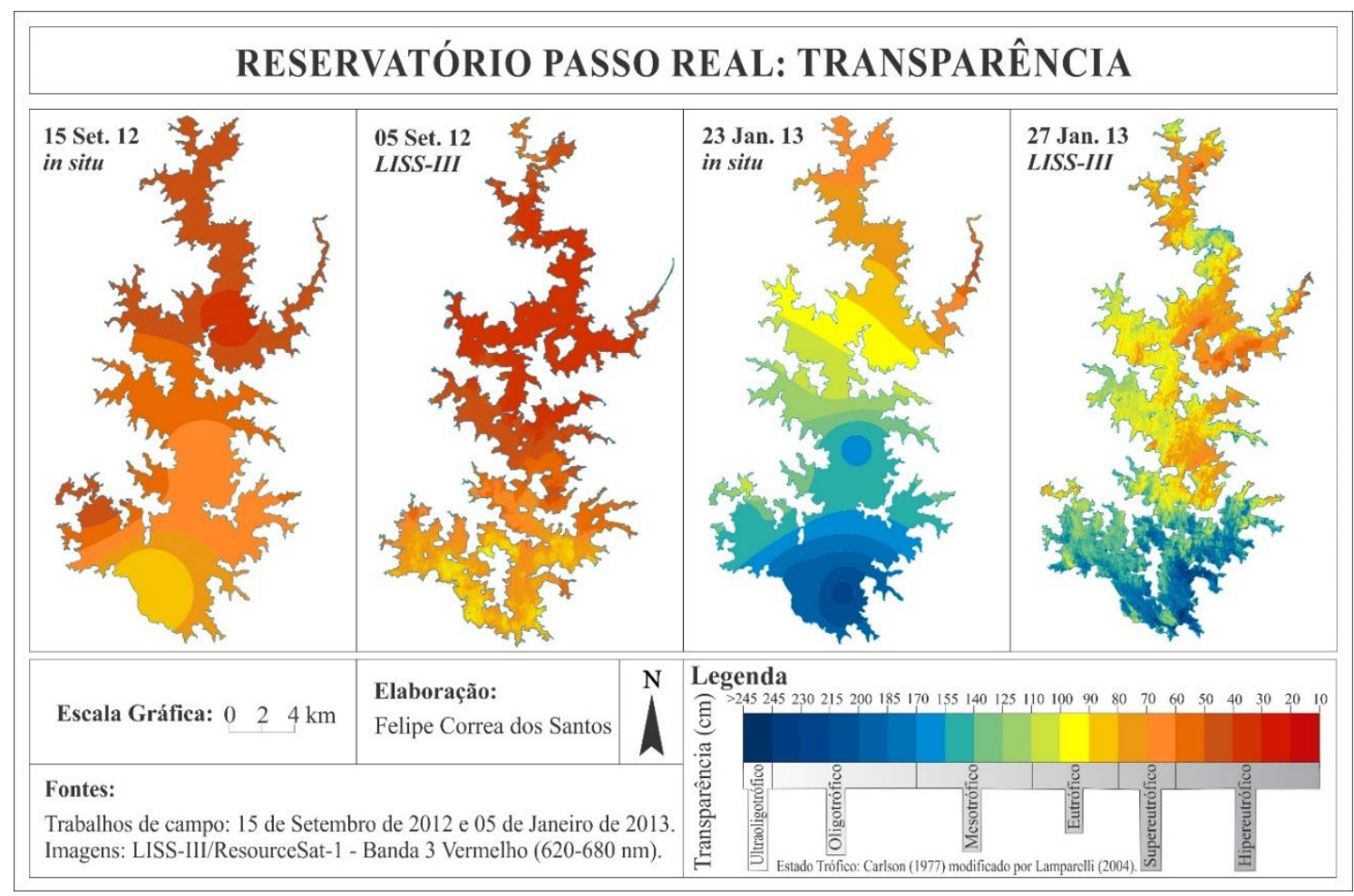

A transparência da água estimada a partir da imagem de satélite apresentou alta semelhança com a transparência da água medida in situ. Em ambos os métodos de obtenção de dados observa-se que mês de setembro de 2012 foi o de menor transparência comparado à janeiro de 2013. Os registros de janeiro de 2013 apontaram maior transparência e maior gradiente longitudinal desta variável, apresentando desde baixas transparências da água no setor de entrada do rio Jacuí até altas transparências no setor próximo ao dique.

Da mesma forma que ocorreu com os dados do total de sólidos em suspensão, ao observar os registros de transparência da água no setor central do reservatório obtidos na imagem de 27 de janeiro de 2013, notase que não há coincidência exata com a espacialização desta variável obtida em campo no dia 23 de janeiro de 2013. 
As concentrações de clorofila- $a$ foram correlacionadas com a razão entre as bandas 4 e 3 (infravermelho próximo/vermelho) do sensor LISS-III. O modelo de regressão apresentou coeficiente de determinação superior a 0,93, considerado de intensidade muito forte e significativo com 95\% de confiança. O modelo de regressão apresentou ajuste linear dos dados e a equação matemática que explica o relacionamento é apresentada na Figura 8.

Figura 8. Diagrama de dispersão entre a variável clorofila-a da água e a razão de bandas espectrais (Banda4/Banda3) do sensor LISSIII/ResourceSat-1.

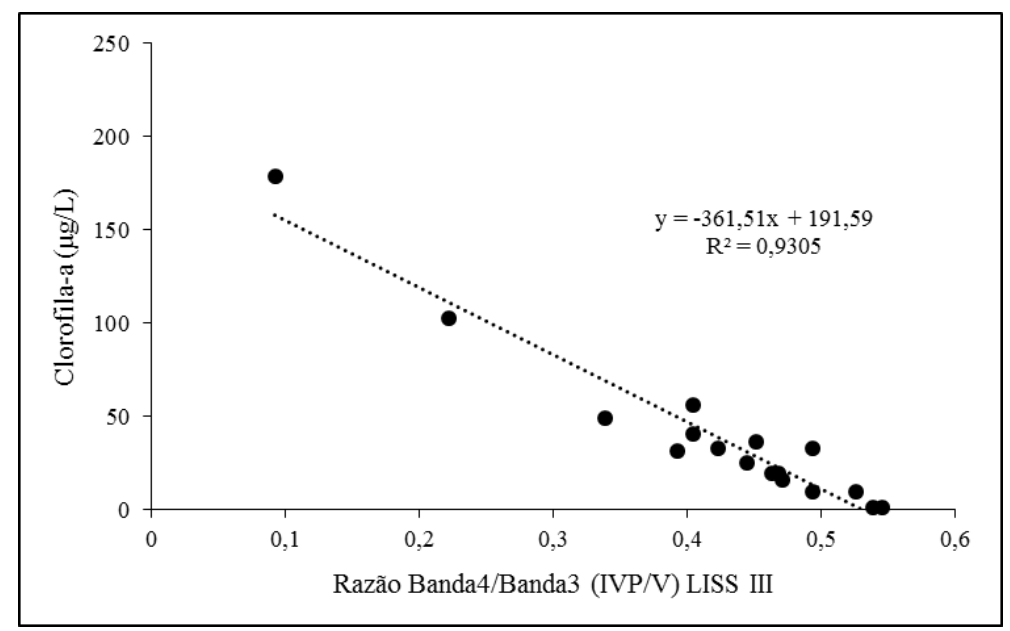

A Figura 9 apresenta a espacialização da clorofila- $a$ obtida in situ no reservatório Passo Real e a estimada pela razão de bandas 4/3 (infravermelho próximo/vermelho) do sensor LISS-III/ResourceSat-1. Na análise da espacialização obtida in situ se observa que altas concentrações de clorofila- $a$ foram registradas nas duas datas analisadas, 15 de setembro de 2012 e 23 de janeiro de 2013. Em setembro de 2012 os altos valores de concentração de clorofila- $a$ foram registrados no setor central até o dique do reservatório e em janeiro de 2013 ocorreu desde o setor de entrada do rio Jacuí-Mirim até o setor central do reservatório. As concentrações de clorofila-a registradas no reservatório em janeiro de 2013 foram mais elevadas que as de setembro de 2012 e de maior variação entre os dados.

Ao analisar a espacialização da clorofila-a obtida por imagem de satélite se observa uma homogeneidade nas altas concentrações de clorofila- $a$ não definindo os diferentes setores do ambiente aquático observados na espacialização dos dados obtidos in situ. Os dados nestes meses foram muito homogêneos, e apesar do modelo de regressão apresentar coeficiente de determinação significativo e considerado de intensidade muito forte. Acredita-se que com dados mais diversificados e com amplitude maior de dados de clorofila- $a$ a estimativa seria mais consistente com os dados obtidos in situ. 
Figura 9. Espacialização da variável clorofila-a obtida in situ no reservatório Passo Real e estimada pela razão de bandas espectrais (Banda4/Banda3) do sensor LISS-III/ResourceSat-1.

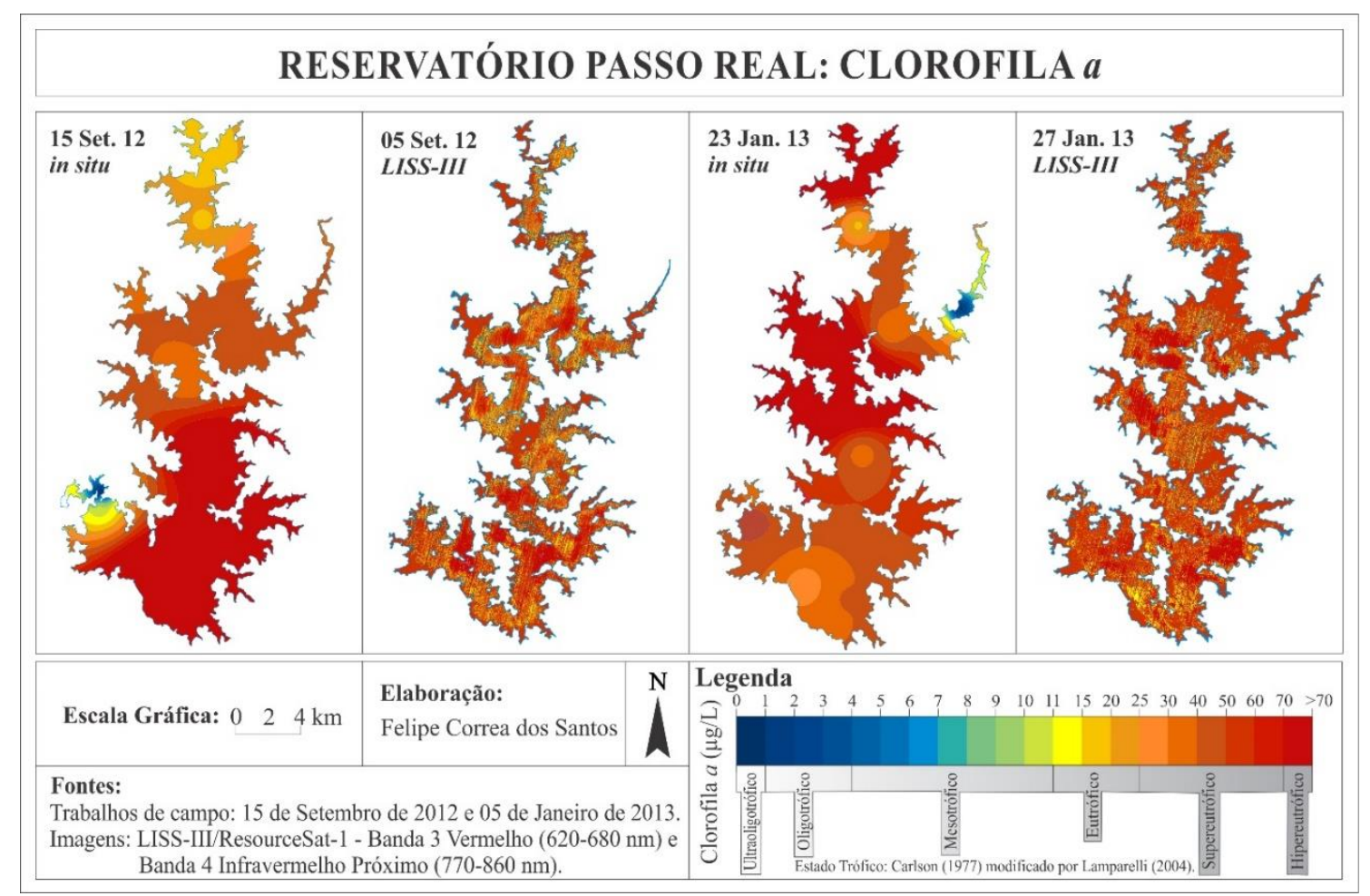

Diversos estudos exploraram a possibilidade de determinar a qualidade da água por sensoriamento remoto e muitos desses estudos consideram as bandas das regiões espectrais referentes ao infravermelho próximo e ao vermelho como satisfatórias para o monitoramento de concentrações de clorofila-a (THIEMANN; KAUFMANN, 2000; SHEELA et al, 2012; AVDAN et al, 2019).

\section{CONSIDERAÇÕES FINAIS}

Os resultados obtidos confirmam o pressuposto inicial do estudo de que as bandas espectrais de imagens LISS-III/Resourcesat-1 tem potencial para estimar concentrações de constituintes opticamente ativos presentes no reservatório Passo Real.

O estudo apresentou metodologias relacionadas ao sensoriamento remoto para a caracterização de variáveis limnológicas e com sua aplicação foi possível identificar e mapear constituintes opticamente ativos do ambiente aquático. A partir do conjunto de dados disponível foi possível produzir modelos capazes de estimar o total de sólidos em suspensão, a turbidez e a transparência da água com apenas uma única banda espectral, a banda 3 do sensor LISS-III/Resourcesat-1. Com a aplicação da técnica de razão de bandas espectrais foi possível gerar um modelo para estimativa da concentração de clorofila- $a$. 
Os modelos de regressão referentes aos dados de turbidez e transparência da água apresentaram coeficiente de determinação de intensidade muito forte e forte, respectivamente. Os modelos para estimativa do total de sólidos em suspensão apresentou coeficiente de determinação regular e resultou em estimativas que se aproximaram com dados obtidos em campo, porém é possível notar algumas divergências em alguns setores do reservatório. A variável clorofila-a apresentou semelhança entre os dados estimados e os dados obtidos in situ, porém os dados estimados não apresentaram setores bem definidos do reservatório como foi possível identificar nos dados in situ. Acredita-se que este fato tenha acontecido pela homogeneidade nas altas concentrações de clorofila- $a$, necessitando-se de uma amplitude maior nos dados para a construção de um modelo mais eficiente, caso necessário.

Com a obtenção de informações relacionadas com a presença e concentração de constituintes opticamente ativos da água e a possibilidade de monitoramento do ambiente aquático utilizando produtos e técnicas de sensoriamento remoto orbital, espera-se que esse modelo possa ser aplicado em cenários de diferentes datas. O ajuste mostrou-se eficiente para a inferência de constituintes opticamente ativos presentes na área de estudo. Embora não tenha sido validado adverte-se que esta etapa é de fundamental importância para que o modelo possa ser aplicado em outras datas.

\section{REFERÊNCIAS}

AGHIGHI, H. et al. Estimation of Water Turbidity in Gorgan Bay, South-East of Caspian Sea by Using IRS-LISS-III Images. Pakistan Journal of Biological Sciences. v. 11, n. 5, 2008, p. 711-718.

APHA - American Public Health Association. Standard Methods for the Examination of Water and Wasterwater. 21 ed. Sprimgfield: Byrd Prepress, 2005.

AVDAN, Z. Y. et al. Monitoring the Water Quality of Small Water Bodies Using High-Resolution Remote Sensing Data. International Journal of Geo-Information. v. 8, n. 553, 2019.

BARBOSA, C. C. F. Sensoriamento remoto da dinâmica da circulação da água do sistema planície de Curuai/Rio Amazonas. 2007. 282p. Tese (Doutorado em Sensoriamento Remoto) - Instituto Nacional de Pesquisas Espaciais, 2005.

BERRA, E. F.; et al. Comparação cruzada dos sensores LISS-III/ResourceSat-1 e TM/Landsat 5. In: Simpósio Brasileiro de Sensoriamento Remoto, 2013, Foz do Iguaçu, Anais... Foz do Iguaçu: Instituto Nacional de Pesquisas Espaciais, 2013. p.9151-9158.

BRANDO, V. E.; DEKKER, A. G. Satellite hyperspectral remote sensing for estimating estuarine and coastal water quality. IEEE Trans. Geosciense and Remote Sensing. vol. 41, no. 6, 2003, p. 1378-1387.

CARLSON, R. E. A trophic state index for lakes. Limmolology Oceanography, v .22, 1977. p. 361-380.

CHANDER, G. Overview of the Resourcesat-1 (IRS-P6). Disponivel em: <http://calval.cr.usgs.gov/documents/IRSP6.pdf> . Acesso em: 09 de julho de 2016.

CHAVEZ JUNIOR, P. S.; An Improved dark-object subtraction technique for atmospheric scattering correction of multispectral data. Remote Sensing of Environment, v. 24, 1988. p. 459-479.

CHOUBEY, V. K.; SUBRAMANIAN, V. Estimation of suspended solids using Indian Remote Sensing Satellite-IA data: a case study from Central India. International Journal of Remote Sensing. v. 13, n.8, 1992, p. 1473-1486.

CHORUS, I.; BARTRAM, J. Water Resources. In: Chorus, I. \& Bartram, J. (Eds). Toxic Cyanobacteria in Water: A guide to their Public Health Consequences, Monitoring and Management. New York, E \& FN Spon, 1999. p. 13-16. 
ENNES, J. Potencial das imagens hiperespectrais orbitais na detecção de componentes opticamente ativos no reservatório de Itupararanga. 125f. Dissertação (Mestrado em Ciências Cartográficas) - Universidade Estadual Paulista, Presidente Prudente, 2008.

FOLKESTAD, A., et al. Inter-comparison of ocean colour data products during algal blooms in the Skagerrak. International Journal of Remote Sensing. vol. 28, no. 3, 2007, p. 569-592.

GIANASI, B. L. et al. Caracterização Espectral da Água e da Vegetação Aquática Submersa no Estuário da Lagoa dos Patos (RS, Brasil). In: Simpósio Brasileiro de Sensoriamento Remoto, 2011, Curitiba, Anais... Curitiba: Instituto Nacional de Pesquisas Espaciais, 2011. p.7051 - 7058.

GOODIN, D. G., et al. A. Analysis of suspended solids in water using remotely sensed high resolution reflectance spectra. Photogrammetric Engineering and Remote Sensing, v. 59, n. 4, 1993. p. 505-510.

HAN, L. Spectral Reflectance with Varying Suspended Sediment Concentration in Clear and Algae-Laden Waters. Photogrammetric Engineering and Remote Sensing, 62, 1997, p. 701-705.

HAN, L.; RUNDQUIST, D. C. Comparison of NIR/RED ratio and first derivative of reflectance in estimating algal-chlorophyll concentration: a case study in a turbid reservoir. Remote Sensing of Environment, 62, 1997, p. 253-261.

HELLWEGER, F. L. et al. Use of satellite imagery for water quality studies in New York Harbor. Estuarine, Coastal and Shelf Science. v.61, 2004, p. 437-448.

JENSEN, J. R. Sensoriamento remoto do ambiente: uma perspectiva em recursos naturais. São José dos Campos, SP: Parêntese, 2009.

KAMPEL, M.; NOVO, E. M. L. M. O sensoriamento remoto da cor da água. In: R. B. SOUZA (ed.), Oceanografia por Satélites, São Paulo: Oficina de Textos, 2005. p.179-196.

KIRK, J. T. O. Ligth and photosynthesis in aquatic ecosystems. 2ed. Cambridge University Press: New York, 1994.

KUTSER, T. Quantitative detection of chlorophyll in cyanobacterial blooms by satellite remote sensing. Limnology and Oceanography. vol. 49, no. 6, 2004, p. 2179-2189.

LAMPARELLI, M. C. Grau de trofia em corpos d'água do estado de São Paulo: avaliação dos métodos de monitoramento. 238f. Tese (Doutorado em Ecologia Aplicada) - Universidade de São Paulo, Instituto de Biociências, São Paulo, 2004.

LOBO, F. L. et al. Mapping potential cyanobacterial bloom using Hyperion/EO-1 data in Patos Lagoon estuary. Acta Limnologica Brasiliensis, vol. 21, no. 3, 2009, p. 299-308.

LOBO, F.L. et al. Time-series analysis of Landsat-MSS/TM/OLI images over Amazonian waters impacted by gold mining activities. Remote Sensing of Environment. v. 157, 2015, p. 170-184.

MACKINNEY, G. Absorption of light by chlorophyll solutions. The Journal of Biological Chemistry. v. 140, 1941. p. $315-322$.

MCCULLOUGH, I. M. Remote Estimation of Regional Lake Clarity with Landsat TM and MODIS Satellite Imagery. 2012. 90f. Dissertação (Mestrado em Ecologia e Ciências Ambientais) - The University of Maine, Orono, 2012.

MARKHAM, B. L.; BARKER, J. L.; Landsat MSS and TM post-calibration dynamic ranges, exoatmospheric reflectances and at-satellite temperature. EOSAT Landsat Technical Notes, № 1, August, 1986. 8p.

MOBLEY, C. D. Light and water: radiative transfer in natural waters. San Diego: Academic Press, 1984.

MOTA, S. Introdução à Engenharia Ambiental. Rio de Janeiro: ABES, 1997. 292 p.

NOVO, E. M. L. de M. et al. Sistemas aquáticos continentais. In: RUDORFF, F. T. et al. O Sensor Modis e suas aplicações ambientais no Brasil. São José dos Campos: Parêntese, 2007.

NRSA - National Remote Sensing Agency. Resourcesat-1 Data User's Handbook. Department of space govt. of India, 2004. 137p.

PARANHOS, R. Alguns métodos para análise de água. Rio de Janeiro, UFRJ, Sub-Reitoria de Ensino de Graduação e Corpo Discente/SR1, 1996, 200p. (Cadernos didáticos).

RUDORFF, C. M. Estudo da composição das águas da Planície Amazônica por meio de dados de reflectância do sensor Hyperion/EO-1 e de espectrômetro de campo visando à compreensão da variação temporal dos seus constituintes opticamente ativos. 2006. 140f. Dissertação (Mestrado em Sensoriamento remoto) - Instituto Nacional de Pesquisas Espaciais, São José dos Campos, 2006.

RUNDQUIST, D. C. et al. Remote Measurement of Algal Chlorophyll in Surface Waters: The Case for the First Derivative of Reflectance Near 690 nm. Photogrammetric Engineering \& Remote Sensing, vol. 62, no. 2, 1996, p. 195-200. 
SHEELA, A. M. et al. (2010). Trophic state index of a lake system using IRS (P6-LISS III) satellite imagery. Environmental Monitoring and Assessment, v. 777, n. 1-4, 2010, p. 575-592.

SHEELA, A. M. et al. Computation of physical characteristics of a lake system using IRS P6 (LISS-III) imagery. International Journal of Applied Earth Observation and Geoinformation. v. 14, n. 1, 2012, p. 221-232.

SILVA, C. B.; et al. Comparação entre dados dos sensores LISS-III/Resourcesat-1 e ETM+/Landsat 7. In: Simpósio Brasileiro de Sensoriamento Remoto, 2013, Foz do Iguaçu, Anais... Foz do Iguaçu: Instituto Nacional de Pesquisas Espaciais, 2013. p.9138- 9142.

SOMVANSHI, S.; et al. Integrated remote sensing and GIS approach for water quality analysis of Gomti river, Uttar Pradesh. International Journal of Environmental. v.3, n.1, 2012. p.62-74.

THIEMANN, S.; KAUFMANN, H. Determination of Chlorophyll Content and Trophic State of Lakes Using Field Spectrometer and IRS-1C Satellite Data in the Mecklenburg Lake District, Germany. Remote Sensing of Environment. v.73, n.1, 2000. p.227-235.

TONIOLO, G. R. et al. Análise temporal da relação entre a reflectância da água e o uso e cobertura da terra no reservatório Passo Real no Alto Jacuí - RS. Relatório Técnico apresentado ao Conselho Nacional de Desenvolvimento Cientifico e Tecnológico (CNPq) processo GAP/UFSM no 032117 como comprovante das atividades referentes ao projeto PIBIC/CNPq/UFSM, 2012.

VALERIO, A. M.; KAMPEL, M. Uso de imagens LISS-III para a caracterização espectral da pluma do Rio Paraíba do Sul. In: Simpósio Brasileiro de Sensoriamento Remoto, 2013, Foz do Iguaçu, Anais... Foz do Iguaçu: Instituto Nacional de Pesquisas Espaciais, 2013. p. 5353-5360.

YUNES, J. S.; ARAÚJO, E. A. C. Protocolo para análise de clorofila-a na água. Rio Grande: Unidade de Pesquisa em Cianobactérias da Fundação Universidade Federal de Rio Grande. [s/d].

WANI, M. M. et al. Quantification of suspended solids in Dal lake, Srinagar using remote sensing technology. Journal of the Indian Society of Remote Sensing. v. 24, 1996, p. 25-32. 